\title{
Traversable wormholes with vanishing sound speed in $f(R)$ gravity
}

\author{
Salvatore Capozziello ${ }^{1,2,3,4, \mathrm{a}}{ }_{\mathbb{D}}$, Orlando Luongo ${ }^{5,6,7, \mathrm{~b}}$, Lorenza Mauro ${ }^{8,9, \mathrm{c}}$ \\ ${ }^{1}$ Dipartimento di Fisica "E. Pancini”, Università di Napoli "Federico II", Via Cinthia, 80126 Napoli, Italy \\ 2 Scuola Superiore Meridionale, Largo S. Marcellino 10, 80138 Napoli, Italy \\ 3 Istituto Nazionale di Fisica Nucleare (INFN), Sezione di Napoli, Via Cinthia, Napoli, Italy \\ ${ }^{4}$ Laboratory of Theoretical Cosmology, Tomsk State University of Control Systems and Radioelectronics \\ (TUSUR), Tomsk, Russia \\ 5 Physics Division, University of Camerino, Via Madonna delle Carceri, Camerino, Italy \\ 6 Dipartimento di Matematica, Università di Pisa, Largo Bruno Pontecorvo, Pisa, Italy \\ 7 National Nanotechnology Laboratory of Open Type (NNLOT), \\ Al-Farabi Kazakh National University, Al-Farabi av. 71, 050040 Almaty, Kazakhstan \\ 8 Dipartimento di Matematica e Fisica, Università degli Studi "Roma Tre", Via della Vasca Navale, Rome, \\ Italy \\ ${ }^{9}$ Istituto Nazionale di Fisica Nucleare, Sezione di Frascati, Via Enrico Fermi, Rome, Italy
}

Received: 27 December 2020 / Accepted: 11 January 2021

(C) The Author(s) 2021

\begin{abstract}
We derive exact traversable wormhole solutions in the framework of $f(R)$ gravity with no exotic matter and with stable conditions over the geometric fluid entering the throat. For this purpose, we propose power-law $f(R)$ models and two possible approaches for the shape function $b(r) / r$. The first approach makes use of an inverse power-law function, namely $b(r) / r \sim r^{-1-\beta}$. The second one adopts Padé approximants, used to characterize the shape function in a model-independent way. We single out the $P(0,1)$ approximant where the fluid perturbations are negligible within the throat, if the sound speed vanishes at $r=r_{0}$. The former guarantees an overall stability of the geometrical fluid into the wormhole. Finally, we get suitable bounds over the parameters of the model for the above discussed cases. In conclusion, we find that small deviations from general relativity give stable solutions.
\end{abstract}

\section{Introduction}

Wormholes are solutions of field equations in General Relativity (GR) and in several theories of gravity. They can be interpreted as "short-cuts" connecting different space-time regions [1] and so they represent hypothetical tunnels between two asymptotic regimes of the same space time [2]. In the most accredited scenario, the wormhole shortcut path is traversable through a minimal surface area called wormhole throat. The simplest approach showing these features is the so-called Einstein-Rosen bridge coming from the connection of two Schwarzschild solutions [3-6]. It is characterized by spherical symmetry and by the presence of an event horizon. This implies that any observer, trying to cross the wormhole throat, inevitably falls

\footnotetext{
a e-mail: capozziello@na.infn.it (corresponding author)

b e-mail: orlando.luongo@unicam.it

c e-mail: lorenza.mauro@lnf.infn.it
} 
into the singularity [7]. Hence, the metric itself a priori prevents the traversability due to the singularity. Consequently, to heal this issue, one can consider non-singular metrics defined for every radial coordinates [8]. However, if the Birkhoff theorem is valid and matter fields are included in a non-vanishing energy-momentum tensor, this approach leads to severe bounds at the wormhole throat. There, the condition $\tau_{0}>\rho_{0} c^{2}$ must hold, i.e., radial tension might be large enough to exceed the total mass-energy density [8]. Consequently the energymomentum tensor violates the null energy condition (NEC) at the throat $[1,9], T_{\mu \nu} k^{\mu} k^{\nu}<0$, in naive analogy to some cosmological contexts, see e.g., [10,11]. Thus, in this standard approach, i.e., in the framework of GR, one is forced to take a negative energy density and pressure. This exotic landscape provides a structure that can be traversable, albeit it is not clear how matter could exhibit negative energy density and pressure [12]. In other words, standard matter cannot be used to achieve stable wormhole solutions in the framework of GR.

In this respect, several approaches have been proposed to alleviate the problem. They focus mainly in considering exotic forms of matter to overcome this strange behavior. Conversely, extended and/or modified theories of gravity are natural suites where this can be addressed retaining standard matter [13-17]. In fact, in these scenarios, the above conditions do not apply directly to matter and so, in lieu of imposing exotic conditions over the energy and pressure, one can take geometry to play the role of exotic matter [18]. This mimics the wormhole properties through higher-order curvature terms and/or effective field theories that can be mapped into Lagrangians extending the Hilbert-Einstein one.

In this paper, in analogy to GR, we consider a spherically symmetric metric with two asymptotically flat regions. In particular, we take in to account $f(R)$ gravity theories, in metric formalism, and we assume time-independent metric coefficients, using the widelyconsolidate Morris-Thorne space time. We thus determine exact solutions of traversable wormhole, without violating the signs of energy and pressure, postulating a power-law form for $f(R)$, i.e., $f(R)=f_{0} R^{1+\epsilon}$ where $\epsilon$ is a real number. Immediately, GR can be recovered in the limit $\epsilon \rightarrow 0$. In this perspective, we can control deviations from GR and the role of geometric terms in stabilizing the wormhole solutions.

In this context, two classes of stable and traversable wormhole can be recovered.

In the first case, the throat is assumed as an inverse power of the radial coordinate. In the second case, we consider a parameter $\alpha$ controlling the size of the throat. It is worth noticing that these solutions can be recovered by assuming simple rational series, made in terms of $(0,1)$ Padé polynomials. We thus provide a physical interpretation over these choices and investigate the physical properties associated with them. In this regard, we impose the fluid perturbations passing through the throat are negligibly small. This condition is achieved if the sound speed is vanishing during the fluid evolution. This feature cannot be found in GR, albeit it gets suitable constraints over the free coefficients of our wormhole picture. In particular, we show that if the sound speed is zero to guarantee stability, even the Starobinsky scalaron [19] (with $\sim R^{2}$ ) is not fully recovered within this scheme, leading to solutions with $\epsilon \neq 0$. It means that GR cannot be trivially recovered by construction. We thus discuss which power law intervals are allowed under this scheme and discuss the corresponding physical implications.

The layout of the paper is the following. In Sect. 2, we take into account $f(R)$ gravity with spherical symmetry assuming a Morris-Thorne metric. In the same section, we introduce the basic ingredients of our approach. Thus, we consider power law $f(R)$ considering deviations with respect to GR. In particular, we impose the form of the metric functions and introduce the Padé expansion. In Sect. 3, the stability condition, given by the vanishing sound speed, is discussed: we find traversable and stable wormholes and constrain the parameters $r_{0}, \beta$ 
of the exact solutions and $\epsilon$ of the related gravity model. Discussion and conclusions are reported in Sect. 4.

\section{$2 f(R)$ wormholes with rational shape function}

Extended theories of gravity are built up through effective Lagrangians and can present higher-order terms in curvature invariants that could mimic the exotic behavior of matter within a wormhole [14,20]. In principle, they are extensions of Einstein theory where GR is a particular case or it is recovered as soon as higher-order terms reduce to $R$. Here, we consider a straightforward extension which is $f(R)$ gravity in the metric formalism [14,21], that is $S=\int d^{4} x \sqrt{-g}[f(R)+\mathcal{L}]$ where $f(R)$ is a generic function of the curvature scalar $R, g$ is the determinant of the metric tensor and $\mathcal{L}$ is the Lagrangian of standard matter minimally coupled to gravity.

Varying with respect to the metric implies the following fourth-order field equations $[22,23]$ :

$$
G_{\mu \nu}=\frac{1}{f_{R}(R)}\left\{\frac{1}{2} g_{\mu \nu}\left[f(R)-R f_{R}(R)\right]+f_{R}(R)_{; \mu \nu}-g_{\mu \nu} \square f_{R}(R)\right\}+\frac{T_{\mu \nu}^{(m)}}{f_{R}(R)},
$$

where $T_{\mu \nu}^{(m)}$ is the stress-energy tensor of ordinary matter. The right-hand side of (1) can be regarded as an effective stress-energy tensor $T_{\mu \nu}^{(e f f)}$, given by the sum of $T_{\mu \nu}^{(m)}$ and a curvature fluid energy-momentum tensor $T_{\mu \nu}^{(\text {(curv) }}$, sourcing the effective Einstein equations. ${ }^{1}$

A static and spherically symmetric wormhole solution is

$$
d s^{2}=e^{2 \Phi(r)} \mathrm{d} t^{2}-\frac{1}{1-b(r) / r} d r^{2}-r^{2} \mathrm{~d} \Omega^{2},
$$

which is the so-called Morris and Thorne metric [8]. Equation (2) characterizes a wormhole with the following features: (i) the space-time is static and spherically symmetric; (ii) the throat has a minimal surface connecting two asymptotically flat regions; (iii) there is no Killing horizon and then two-way travels are enabled. The physical realization of such criteria depends on the gravitational forces, the proper time for crossing and astrophysical scales where possible wormholes are expected [26].

In this picture, $b(r)$ and $\Phi(r)$ are functions of the radial coordinate and they are denoted respectively as shape and redshift functions. The radial coordinate $r$ ranges from a minimum and a positive value $r_{0}$, defining the wormhole throat, to infinity. In order to avoid the presence of event horizons, one imposes that $\Phi(r)$ is finite at any $r$. It is possible to construct asymptotically flat space times, where $b(r) / r \rightarrow 0$ and $\Phi \rightarrow 0$ as $r \rightarrow \infty$. A fundamental ingredient in wormhole physics is the the so-called flare-out condition of the wormhole throat $b\left(r_{0}\right)=r_{0}$ [8] given by the condition $\left(b^{\prime} r-b\right) / 2 b^{2}<0$. In GR, the latter condition implies that through the Einstein field equation, the stress-energy tensor violates the NEC at the throat, i.e., $\left.T_{\mu \nu} k^{\mu} k^{\nu}\right|_{r_{0}}<0$.

In this paper, we consider:

$$
2 \Phi(r)=\frac{r_{0}}{r}
$$

\footnotetext{
1 This interpretation is based on the fact that further degrees of freedom coming from higher-order gravity can be recast as an effective perfect fluid which reduces to the standard energy-matter one as soon as GR is recovered. For a rigorous demonstration, see [24,25].
} 
that resembles a Newtonian potential for $\Phi(r)$ in analogy to black hole physics [27,28]. The ratio $\frac{b(r)}{r}$ is debated and its form is a priori unknown. Finding out the forms of $\Phi(r)$ and $b(r)$ from the field equations means to derive a Morris-Thorne-like wormhole solution. Here, we follow two physically motivated strategies imposing:

$$
\begin{aligned}
\frac{b(r)}{r} & =\left(\frac{r_{0}}{r}\right)^{1+\beta}, \\
\frac{b(r)}{r} & =\frac{r_{0}}{1+\alpha r},
\end{aligned}
$$

with $\beta \in \mathbb{R}$ and $\beta+1>0$ and $\alpha \equiv \frac{r_{0}-1}{r_{0}}$ to guarantee that at $r=r_{0}$ the wormhole is not singular.

Therefore, our wormhole metric takes two possible forms:

$$
\begin{aligned}
& d s^{2}=e^{r_{0} / r} \mathrm{~d} t^{2}-\frac{1}{1-\left(\frac{r_{0}}{r}\right)^{\beta+1}} d r^{2}-r^{2} \mathrm{~d} \Omega^{2}, \\
& d s^{2}=e^{r_{0} / r} \mathrm{~d} t^{2}-\frac{1}{1-\frac{r_{0}}{1+\alpha r}} d r^{2}-r^{2} \mathrm{~d} \Omega^{2} .
\end{aligned}
$$

In both cases, the expressions are polynomials characterizing $b / r$. To enable stability, one can require that within the whole interval of $r$, the ratio $b(r) / r$ does not diverge. An intriguing proposal has been shown in [28], where a shape function of the type $b(r)=$ $r_{0}\left(\frac{r_{0}}{r}\right)^{\beta} \exp \left(-\delta \frac{r-r_{0}}{r_{0}}\right)$ has been introduced. As $r-r_{0} \ll 1$, it is possible to make a Taylor expansion that leads to Eq. (4a) that turns out to be an extension of the cases discussed in $[27,28]$. Hereafter, Eq. (4a) will be dubbed phenomenological shape function, to stress that is has been argued from heuristic considerations.

At $r=r_{0}$, to avoid discontinuities in the Morris-Thorne metric, we can require the domain to be stable even before $r=r_{0}$. Thus, one can imagine to expand around $r=r_{0}$ in terms of rational expansions, made by Padé functions, widely used in recent literature [16,29,30]. The corresponding ratio, constructed by means of Padé polynomials, changes dramatically the form of solutions or leaves it unaltered. As a prototype of our recipe, we take into account the simplest Padé expansion [31]. To this end, we recall that the Padé technique is built up from the standard Taylor series, being to lower divergences or singular points. Hence, given a function $f(z)=\sum_{i=0}^{\infty} c_{i} z^{i}$, expanded with a given set of coefficients, namely $c_{i}$, it is approximated by means of a $(n, m)$ Padé approximant by the ratio [34]:

$$
P_{n, m}(z)=\frac{\sum_{\kappa=0}^{n} a_{\kappa} z^{\kappa}}{1+\sum_{\sigma=1}^{m} b_{\sigma} z^{\sigma}},
$$

where the Taylor expansion matches the coefficients of the expansion up to the highest possible order:

$$
\begin{aligned}
& P_{n, m}^{\prime}(0)=f^{\prime}(0), \\
& \vdots \\
& P_{n, m}^{(n+m)}(0)=f^{(n+m)}(0),
\end{aligned}
$$

with the additional request $P_{n, m}(0)=f(0)$.

The numerator is thus constructed to have $n+1$ independent coefficients, whereas in the denominator, it is $m$, for a total of $n+m+1$ unknown terms. 
For small radii, rational expressions are essentially indistinguishable from the Taylor one, but, at larger radii, the convergence radius of rational polynomials is determined by the following practical rule $[35,36]$ :

\section{The most suitable rational approximation order leads to the function that maximizes the} convergence radius.

II. The most suitable rational approximation minimizes the involved free constants.

Consequently, a small number of free parameters is essential to enable the rational approximation to be convergent, providing the compromise between arbitrary-order expansions and minimal number of free parameters in the denominator.

The lowest Padé orders are two: $(1,0)$ and $(0,1)$. They turn out to be the simplest approaches to use in the framework of wormholes. We are forced to take the $(0,1)$ order since it guarantees that all the other assumptions over the stability of $b / r$ are preserved. ${ }^{2}$ In both cases, the asymptotic conditions are automatically satisfied, i.e., $e^{r_{0} / r} \rightarrow 1$ and $\frac{1}{1-b(r) / r} \rightarrow 1$ as $r \rightarrow \infty$.

We are now able to get the corresponding energy conditions and to check whether the above consistency conditions are satisfied.

Let us start with the field Eq. (1) which can be rewritten as :

$$
\begin{aligned}
T_{\mu \nu}^{(m)}= & f_{R}(R) G_{\mu \nu}-\left\{\frac{1}{2} g_{\mu \nu}\left[f(R)-R f_{R}(R)\right]\right. \\
& \left.+f_{R}(R)_{; \mu \nu}-g_{\mu \nu} \square f_{R}(R)\right\} .
\end{aligned}
$$

In order to write energy conditions [1,9], we can choose:

$$
T_{\nu}^{\mu}=\operatorname{diag}\left(\rho,-p_{r},-p_{t},-p_{t}\right), \quad f(R)=f_{0} R^{1+\epsilon},
$$

where $p_{t}$ is the tangential pressure, $p_{r}$ the radial pressure and $\rho$ the energy density, whereas $f(R)$ is a power law, with $f_{0}$ dimensional constant. ${ }^{3}$ For $\epsilon \ll 1$, it can be written in the form

$$
f(R) \sim R+\epsilon R \ln R+\mathcal{O}\left(\epsilon^{2}\right),
$$

corresponding to the GR plus a correction. Clearly, this form is useful to control little deviations with respect to the standard Einstein theory. Recently, this approach revealed particularly useful to study compact objects, like neutron stars and black holes, where deviations with respect to GR can be useful to fit observations [32,33].

Here, we adopt a similar approach to investigate which cases correspond to small departures from Einstein's gravity according to the values of $\epsilon$. As we will see below, $\epsilon$ is constrained in range of values providing wormhole solutions with vanishing sound speed. In other words, we can state that:

Stable and traversable wormhole solutions are possible for small deviations of Einstein's gravity in the presence of standard perfect fluid matter.

Starting from (10) and (11), we get the components of the energy-momentum tensor for the generic metric (2):

$$
\rho(r)=\frac{1}{2}\left[\frac{r\left(r_{0}^{2}+r\left(r_{0}-4 r\right) b^{\prime}(r)\right)-r_{0}\left(r_{0}+r\right) b(r)}{2 r^{5}}\right]^{1+\epsilon}
$$

\footnotetext{
2 The expansion $(1,0)$ corresponds to a first-order Taylor expansion and does not work well to guarantee that, for $r \rightarrow \infty, \frac{b}{r} \rightarrow 0$. We have to check this property for all cases at the wormhole throat, namely $r_{0}$, $b\left(r_{0}\right) / r_{0}=1$.

${ }^{3}$ From now on, we set this constant equal to 1 .
} 


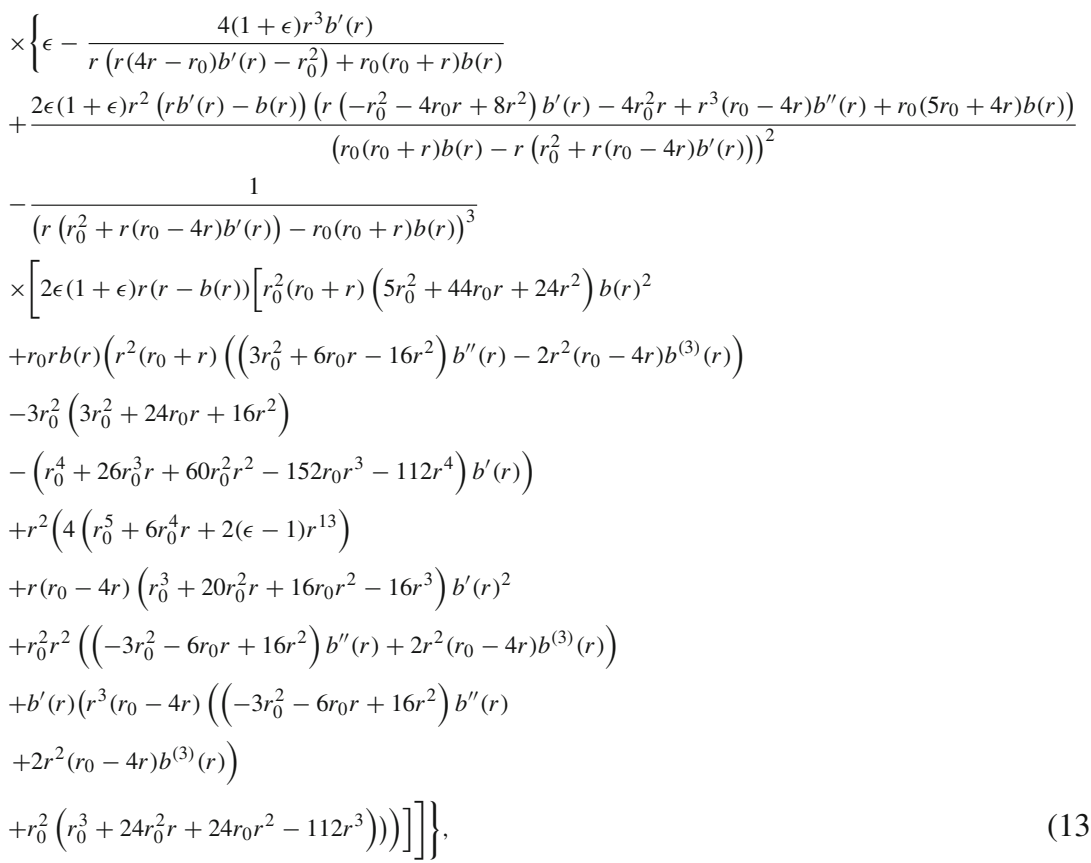

$$
\begin{aligned}
p_{r}(r)= & \frac{1}{2}\left[\frac{r\left(r_{0}^{2}+r\left(r_{0}-4 r\right) b^{\prime}(r)\right)-r_{0}\left(r_{0}+r\right) b(r)}{2 r^{5}}\right]^{1+\epsilon} \\
& \times\left\{\left[-\epsilon+\frac{4(1+\epsilon) r\left(\left(r-r_{0}\right) b(r)+r_{0} r\right)}{r\left(r\left(4 r-r_{0}\right) b^{\prime}(r)-r_{0}^{2}\right)+r_{0}\left(r_{0}+r\right) b(r)}\right.\right. \\
& +\left(2 \epsilon ( 1 + \epsilon ) r ( r _ { 0 } - 4 r ) ( r - b ( r ) ) \left(r\left(r_{0}^{2}+4 r_{0} r-8 r^{2}\right) b^{\prime}(r)\right.\right. \\
& \left.\left.\left.+4 r_{0}^{2} r+r^{3}\left(4 r-r_{0}\right) b^{\prime \prime}(r)-r_{0}\left(5 r_{0}+4 r\right) b(r)\right)\right)\right] \\
& \left.\times\left[\left(r_{0}\left(r_{0}+r\right) b(r)-r\left(r_{0}^{2}+r\left(r_{0}-4 r\right) b^{\prime}(r)\right)\right)^{2}\right]^{-1}\right\},
\end{aligned}
$$

$$
\begin{aligned}
p_{t}(r)= & \frac{1}{2}\left[\frac{r\left(r_{0}^{2}+r\left(r_{0}-4 r\right) b^{\prime}(r)\right)-r_{0}\left(r_{0}+r\right) b(r)}{2 r^{5}}\right]^{1+\epsilon} \\
& \times\left\{\frac{(1+\epsilon)\left(\left(r_{0}^{2}+r_{0} r+2 r^{2}\right) b(r)+r\left(r\left(r_{0}-2 r\right) b^{\prime}(r)-r_{0}\left(r_{0}+2 r\right)\right)\right)}{r\left(r_{0}^{2}+r\left(r_{0}-4 r\right) b^{\prime}(r)\right)-r_{0}\left(r_{0}+r\right) b(r)}+\right. \\
& -\epsilon+\frac{2 \epsilon(1+\epsilon) r^{2}\left(b(r)-r b^{\prime}(r)\right)\left(r\left(-r_{0}^{2}-4 r_{0} r+8 r^{2}\right) b^{\prime}(r)-4 r_{0}^{2} r+r^{3}\left(r_{0}-4 r\right) b^{\prime \prime}(r)+r_{0}\left(5 r_{0}+4 r\right) b(r)\right)}{\left(r_{0}\left(r_{0}+r\right) b(r)-r\left(r_{0}^{2}+r\left(r_{0}-4 r\right) b^{\prime}(r)\right)\right)^{2}} \\
& +\frac{1}{\left(r\left(r_{0}^{2}+r\left(r_{0}-4 r\right) b^{\prime}(r)\right)-r_{0}\left(r_{0}+r\right) b(r)\right)^{3}} \\
& \times\left[2 \epsilon ( 1 + \epsilon ) r ( r - b ( r ) ) \left[r_{0}^{2}\left(r_{0}+r\right)\left(5 r_{0}^{2}+54 r_{0} r+32 r^{2}\right) b(r)^{2}\right.\right. \\
& +r_{0} r b(r)\left(-r_{0}^{2}\left(9 r_{0}^{2}+90 r_{0} r+64 r^{2}\right)\right. \\
& -\left(r_{0}^{4}+28 r_{0}^{3} r+80 r_{0}^{2} r^{2}-192 r_{0} r^{3}-160 r^{4}\right) b^{\prime}(r)
\end{aligned}
$$

\section{를 Springer}




$$
\begin{aligned}
& \left.+r^{2}\left(r_{0}+r\right)\left(\left(3 r_{0}^{2}+8 r_{0} r-24 r^{2}\right) b^{\prime \prime}(r)-2 r^{2}\left(r_{0}-4 r\right) b^{(3)}(r)\right)\right) \\
& +r^{2}\left(4\left(r_{0}^{5}+8 r_{0}^{4} r+2(\epsilon-1) r^{13}\right)\right. \\
& +r\left(r_{0}-4 r\right)\left(r_{0}+2 r\right)\left(r_{0}^{2}+20 r_{0} r-16 r^{2}\right) b^{\prime}(r)^{2}+r_{0}^{2} r^{2} \\
& \times\left(\left(-3 r_{0}^{2}-8 r_{0} r+24 r^{2}\right) b^{\prime \prime}(r)+2 r^{2}\left(r_{0}-4 r\right) b^{(3)}(r)\right) \\
& +b^{\prime}(r)\left(r ^ { 3 } ( r _ { 0 } - 4 r ) \left(\left(-3 r_{0}^{2}-8 r_{0} r+24 r^{2}\right) b^{\prime \prime}(r)\right.\right. \\
& \left.+2 r^{2}\left(r_{0}-4 r\right) b^{(3)}(r)\right) \\
& \left.\left.\left.\left.\left.+r_{0}^{2}\left(r_{0}^{3}+26 r_{0}^{2} r+40 r_{0} r^{2}-160 r^{3}\right)\right)\right)\right]\right]\right\} .
\end{aligned}
$$

Here, the form of $b(r)$ is not specified. If we consider the energy-momentum tensor for perfect fluids written in the form $T^{\mu}{ }_{v}=\operatorname{diag}(\rho,-p,-p,-p)$, the average pressure is $p(r)=\frac{1}{3}\left[p_{r}(r)+2 p_{t}(r)\right]$, and then:

$$
\begin{aligned}
p(r)= & \frac{1}{6}\left[\frac{r\left(r_{0}^{2}+r\left(r_{0}-4 r\right) b^{\prime}(r)\right)-r_{0}\left(r_{0}+r\right) b(r)}{2 r^{5}}\right]^{1+\epsilon} \\
& \times\left\{\frac{4(1+\epsilon) r\left(\left(r-r_{0}\right) b(r)+r_{0} r\right)}{r\left(r\left(4 r-r_{0}\right) b^{\prime}(r)-r_{0}^{2}\right)+r_{0}\left(r_{0}+r\right) b(r)}\right. \\
& -\epsilon+\frac{2 \epsilon(1+\epsilon) r\left(r_{0}-4 r\right)(r-b(r))\left(r\left(r_{0}^{2}+4 r_{0} r-8 r^{2}\right) b^{\prime}(r)+4 r_{0}^{2} r+r^{3}\left(4 r-r_{0}\right) b^{\prime \prime}(r)-r_{0}\left(5 r_{0}+4 r\right) b(r)\right)}{\left(r_{0}\left(r_{0}+r\right) b(r)-r\left(r_{0}^{2}+r\left(r_{0}-4 r\right) b^{\prime}(r)\right)\right)^{2}} \\
& +2\left[-\epsilon+\frac{(1+\epsilon)\left(\left(r_{0}^{2}+r_{0} r+2 r^{2}\right) b(r)+r\left(r\left(r_{0}-2 r\right) b^{\prime}(r)-r_{0}\left(r_{0}+2 r\right)\right)\right)}{r\left(r_{0}^{2}+r\left(r_{0}-4 r\right) b^{\prime}(r)\right)-r_{0}\left(r_{0}+r\right) b(r)}\right. \\
& +\frac{2 \epsilon(1+\epsilon) r^{2}\left(b(r)-r b^{\prime}(r)\right)\left(r\left(-r_{0}^{2}-4 r_{0} r+8 r^{2}\right) b^{\prime}(r)-4 r_{0}^{2} r+r^{3}\left(r_{0}-4 r\right) b^{\prime \prime}(r)+r_{0}\left(5 r_{0}+4 r\right) b(r)\right)}{\left(r_{0}\left(r_{0}+r\right) b(r)-r\left(r_{0}^{2}+r\left(r_{0}-4 r\right) b^{\prime}(r)\right)\right)^{2}} \\
& +\frac{1}{\left(r\left(r_{0}^{2}+r\left(r_{0}-4 r\right) b^{\prime}(r)\right)-r_{0}\left(r_{0}+r\right) b(r)\right)^{3}} \\
& \times\left[2 \epsilon ( 1 + \epsilon ) r ( r - b ( r ) ) \left(r_{0}^{2}\left(r_{0}+r\right)\left(5 r_{0}^{2}+54 r_{0} r+32 r^{2}\right) b(r)^{2}\right.\right. \\
& +r_{0} r b(r)\left(r^{2}\left(r_{0}+r\right)\left(\left(3 r_{0}^{2}+8 r_{0} r-24 r^{2}\right) b^{\prime \prime}(r)-2 r^{2}\left(r_{0}-4 r\right) b^{(3)}(r)\right)\right. \\
& -r_{0}^{2}\left(9 r_{0}^{2}+90 r_{0} r+64 r^{2}\right)+ \\
& \left.-\left(r_{0}^{4}+28 r_{0}^{3} r+80 r_{0}^{2} r^{2}-192 r_{0} r^{3}-160 r^{4}\right) b^{\prime}(r)\right) \\
& +r^{2}\left(4\left(r_{0}^{5}+8 r_{0}^{4} r+2(\epsilon-1) r^{13}\right)\right. \\
& +r\left(r_{0}-4 r\right)\left(r_{0}+2 r\right)\left(r_{0}^{2}+20 r_{0} r-16 r^{2}\right) b^{\prime}(r)^{2}+r_{0}^{2} r^{2} \\
& \times\left(\left(-3 r_{0}^{2}-8 r_{0} r+24 r^{2}\right) b^{\prime \prime}(r)+2 r^{2}\left(r_{0}-4 r\right) b^{(3)}(r)\right) \\
& +b^{\prime}(r)\left(r ^ { 3 } ( r _ { 0 } - 4 r ) \left(\left(-3 r_{0}^{2}-8 r_{0} r+24 r^{2}\right) b^{\prime \prime}(r)\right.\right. \\
& \left.+2 r^{2}\left(r_{0}-4 r\right) b^{(3)}(r)\right) \\
& \left.\left.\left.\left.\left.+r_{0}^{2}\left(r_{0}^{3}+26 r_{0}^{2} r+40 r_{0} r^{2}-160 r^{3}\right)\right)\right)\right]\right]\right\} . \\
&
\end{aligned}
$$

In this way, the null energy condition [1,9] at the throat, for the metric (5), that is for $\frac{b(r)}{r}=\left(\frac{r_{0}}{r}\right)^{\beta+1}$, is expressed as:

$$
\begin{aligned}
\rho+\left.p\right|_{r_{0}}= & \frac{(1+\epsilon)\left(\frac{3 \beta-1}{2 r_{0}^{2}}\right)^{1+\epsilon}\left\{6+\beta-3 \beta^{2}(8+\beta)+(1+\epsilon)[-5+\beta(1+3 \beta(3+\beta))]\right\}}{3(1-3 \beta)^{2}} \\
& \geq 0,
\end{aligned}
$$


while for the metric (6), i.e., for $\frac{b(r)}{r}=\frac{r_{0}}{1+\alpha r}$, it is:

$$
\begin{aligned}
\rho+\left.p\right|_{r_{0}}= & -\frac{(1+\epsilon)\left(-\frac{r_{0}+3}{2 r_{0}^{3}}\right)^{1+\epsilon}\left\{\left(r_{0}-1\right)\left[r_{0}\left(5 r_{0}+9\right)-6\right](1+\epsilon)-2\left[r_{0}\left(3 r_{0}^{2}+r_{0}-15\right)+3\right]\right\}}{3 r_{0}\left(r_{0}+3\right)^{2}} \\
& \geq 0 .
\end{aligned}
$$

Another aspect to check is the flare-out condition [37]:

$$
\left.\frac{b^{\prime}(r) r-b(r)}{2 b(r)^{2}}\right|_{r_{0}}<0,
$$

which becomes, for the metric (5) at the throat:

$$
-\frac{1+\beta}{2 r_{0}}<0 \rightarrow \beta>-1
$$

In the other case, it is fulfilled for:

$$
-\frac{r_{0}-1}{2 r_{0}^{2}}<0 \rightarrow r_{0}>1,
$$

provided that $r_{0}>0$. The consistency of our model is guaranteed in both cases. These results allow to give necessary conditions on the function $b(r) / r$ but they are not sufficient to show that the form of $b(r)$ is of the form of a polynomial. To ensure this hypothesis, we assume that the sound speed, i.e., the variation of the pressure with respect to the density is negligibly small [38]. Combining this additional requirement, we stabilize the solution as we shall show below.

\section{The stability condition and the sound speed}

We require the solutions to be stable, besides being traversable. This reflects to the stability of fluids inside the throat, with the hypothesis of satisfying the above energy conditions.

Thus, let us consider the perturbation condition by means of the adiabatic sound speed, $c_{s}$, i.e., we assume the sound speed definition in adiabatic perturbations, in analogy to what happens in fluid dynamics $[39,40]$. So, defining the adiabatic sound speed by [41-43]:

$$
c_{s}^{2}=\left(\frac{\partial p}{\partial \rho}\right)_{S},
$$

we can guarantee how perturbations affect solutions analyzing its value within the throat. Hence, the sound speed is essential to guarantee the viability of our approximated versions of $b / r$. The above expression for $c_{s}$ can be specified as

$$
\left.\frac{\mathrm{d} p}{\mathrm{~d} \rho}\right|_{r_{0}}=0 .
$$

Plugging Eqs. (13) and (16) in Eq. (23) and considering $\frac{b(r)}{r}=\left(\frac{r_{0}}{r}\right)^{\beta+1}$, we get the following stability condition for the metric (5):

$$
\begin{aligned}
& 147-32 r_{0}^{8} \epsilon(\epsilon-1)(1+\beta)-4(1+\epsilon)^{2}(1+\beta)[5-3 \beta(2+\beta)]^{2} \\
& +\beta\{-428+\beta[-926+3 \beta(148+\beta(161+24 \beta))]\}+ \\
& -(1+\epsilon)\{45+\beta[-288+\beta(-658+3 \beta(60+\beta(95+12 \beta)))]\}
\end{aligned}
$$




$$
\begin{aligned}
& \times\left\{3 \left[16 r_{0}^{8} \epsilon(\epsilon-1)(1+\beta)+2(1+\epsilon)^{2}(1+\beta)[5-3 \beta(2+\beta)]^{2}+\right.\right. \\
& -55+(1+\epsilon)(1+\beta)[5+\beta(-113+3 \beta(-37+\beta(19+6 \beta)))] \\
& +\beta[166-\beta(-400+3 \beta(38+\beta(67+12 \beta)))]]\}^{-1}=0 .
\end{aligned}
$$

Similarly, by considering $\frac{b(r)}{r}=\frac{r_{0}}{1+\alpha r}$, we get the stability condition for the metric (6):

$$
\begin{aligned}
& \frac{1}{3}\left\{-2-r_{0}\left(r_{0}+3\right)\left[r _ { 0 } \left(r_{0}^{2}(35(1+\epsilon)-37)\right.\right.\right. \\
& \left.\left.\quad-12 r_{0}(\epsilon+2)-177(1+\epsilon)+135\right)+90(1+\epsilon)-54\right]\left[r_{0}+36(7-5(1+\epsilon))\right. \\
& \quad+\left(-714+r_{0}\left(393+r_{0}\left(389+r_{0}\left(-137+r_{0}\left(32\left(r_{0}-1\right) r_{0}^{7}-55\right)\right)\right)\right)\right) \\
& \quad+r_{0}(1+\epsilon)\left(354+r_{0}\left(-39+r_{0}(-163\right.\right. \\
& \left.\left.\left.\quad+r_{0}\left(-48 r_{0}^{9}+48 r_{0}^{8}+5 r_{0}+23\right)\right)\right)\right) \\
& \left.\left.\quad+2\left(r_{0}-1\right)(1+\epsilon)^{2}\left(36+r_{0}\left(-108+r_{0}\left(21+r_{0}\left(8 r_{0}^{9}+25 r_{0}+90\right)\right)\right)\right)\right]^{-1}\right\}=0 .
\end{aligned}
$$

We can therefore analyze the consequence of such conditions for our wormhole solutions as reported in the next subsection. The corresponding results are clearly numerical since no analytical solutions can be obtained integrating the above stability conditions ${ }^{4}$ coming from $c_{s}=0$.

\subsection{The wormhole solutions}

In the case of metric (5), we have determined a class of wormhole solutions satisfying the three above conditions, namely the null energy condition (17), the flare-out condition (20) and the stability condition (24). In particular, once the value of the $\beta$ parameter is fixed, we determine the wormhole throat (as a function of $\epsilon$ ) and the values of $\epsilon$ that satisfy Eqs. (17), (20), (24) and the condition $r_{0}>0$. We have summarized the results obtained in Table 1.

In particular, we note that $\epsilon=\frac{1}{2}$, i.e., $f(R)=R^{3 / 2}$, is obtained in three cases ${ }^{5}$ :

1. $\beta=7 \Rightarrow r_{0} \simeq 3.632$;

2. $\beta=8 \Rightarrow r_{0} \simeq 3.862$;

3. $\beta=9 \Rightarrow r_{0} \simeq 4.078$.

These correspond to three wormhole metrics, respectively:

1. $d s^{2}=e^{r_{0} / r} \mathrm{~d} t^{2}-\frac{1}{1-\left(\frac{r_{0}}{r}\right)^{8}} d r^{2}-r^{2} \mathrm{~d} \Omega^{2}$;

2. $d s^{2}=e^{r_{0} / r} \mathrm{~d} t^{2}-\frac{1}{1-\left(\frac{r_{0}}{r}\right)^{9}} d r^{2}-r^{2} \mathrm{~d} \Omega^{2}$;

3. $d s^{2}=e^{r_{0} / r} \mathrm{~d} t^{2}-\frac{1}{1-\left(\frac{r_{0}}{r}\right)^{10}} d r^{2}-r^{2} \mathrm{~d} \Omega^{2}$.

In the case of metric (6), the validity of NEC (18) points out that $1+\epsilon$ must be an integer. For $\epsilon$ integer and odd, we found no solutions satisfying also the flare-out condition (21).

\footnotetext{
4 The assumption $c_{s}=0$ is also used in astrophysics to guarantee stability of virialized structures. See e.g., [44].

$5 f(R)$ gravity with $1+\epsilon=3 / 2$ is particularly relevant for cosmological and astrophysical applications. It is related to invertible conformal transformation [22]. It allows a curvature interpretation of dark matter phenomena according to MOND [45] and the transition from decelerated to accelerated regimes in cosmology [46].
} 
Table 1 Wormhole solutions for different values of the $\beta$ parameter. Here, we adopted the parameterization given in Eq. (4a)

\begin{tabular}{lll}
\hline$\beta$ & $r_{0}$ & $\epsilon$ \\
\hline 0 & $\frac{1275}{128}$ & $\frac{1}{5}$ \\
1 & No solution & No solution \\
2 & $\sqrt[8]{\frac{5332 \epsilon^{2}+14653 \epsilon+150}{96 \epsilon-96 \epsilon^{2}}}$ & $-2.986 \lesssim \epsilon<-1 \cup 0.965 \lesssim \epsilon<1$ \\
3 & $\sqrt[8]{\frac{2\left(100 \epsilon^{2}+317 \epsilon-14\right)}{(1-\epsilon) \epsilon}}$ & $-3.213 \lesssim \epsilon<-1 \cup 0.800 \lesssim \epsilon<1$ \\
4 & $\sqrt[8]{\frac{89780 \epsilon^{2}+289269 \epsilon-9922}{160(1-\epsilon) \epsilon}}$ & $-3.256 \lesssim \epsilon<-1 \cup 0.689 \lesssim \epsilon<1$ \\
5 & $\sqrt[8]{\frac{15000 \epsilon^{2}+48455 \epsilon-1372}{12(1-\epsilon) \epsilon}}$ & $-3.258 \lesssim \epsilon<-1 \cup 0.607 \lesssim \epsilon<1$ \\
6 & $\sqrt[8]{\frac{540988 \epsilon^{2}+1744781 \epsilon-42194}{224(1-\epsilon) \epsilon}}$ & $-3.249 \lesssim \epsilon<-1 \cup 0.542 \lesssim \epsilon<1$ \\
7 & $\sqrt[8]{\frac{8464 \epsilon^{2}+27216 \epsilon-575}{2(1-\epsilon) \epsilon}}$ & $-3.236 \lesssim \epsilon<-1 \cup 0.489 \lesssim \epsilon<1$ \\
8 & $\sqrt[8]{\frac{1988100 \epsilon^{2}+6370997 \epsilon-119554}{288(1-\epsilon) \epsilon}}$ & $-3.223 \lesssim \epsilon<-1 \cup 0.446 \lesssim \epsilon<1$ \\
9 & $\sqrt[8]{\frac{213160 \epsilon^{2}+680759 \epsilon-11492}{20(1-\epsilon) \epsilon}}$ & $-3.210 \lesssim \epsilon<-1 \cup 0.409 \lesssim \epsilon<1$ \\
\hline
\end{tabular}

The allowed range values of $\epsilon$ are also reported

Table 2 Wormhole solutions for different values of $\epsilon$

\begin{tabular}{ll}
\hline$\epsilon$ & $r_{0}$ \\
\hline 0 & No solution \\
2 & 1.150 \\
4 & 1.114 \\
6 & 1.090 \\
8 & 1.075 \\
10 & 1.064 \\
12 & 1.056 \\
14 & 1.050 \\
16 & 1.045 \\
18 & 1.040
\end{tabular}

Here, we adopted the parameterization given in Eq. (4b)

Consequently, the only solutions that satisfy all three conditions (18), (21) and (25) are those with $\epsilon$ integer and even. Therefore, once the value of $\epsilon$ is fixed, we determine the corresponding wormhole throat. The results are given in Table 2. We note that $\epsilon=0$, implying GR leads to no solutions as the NEC is not satisfied. The theoretical consequences of our approach are summarized below. 

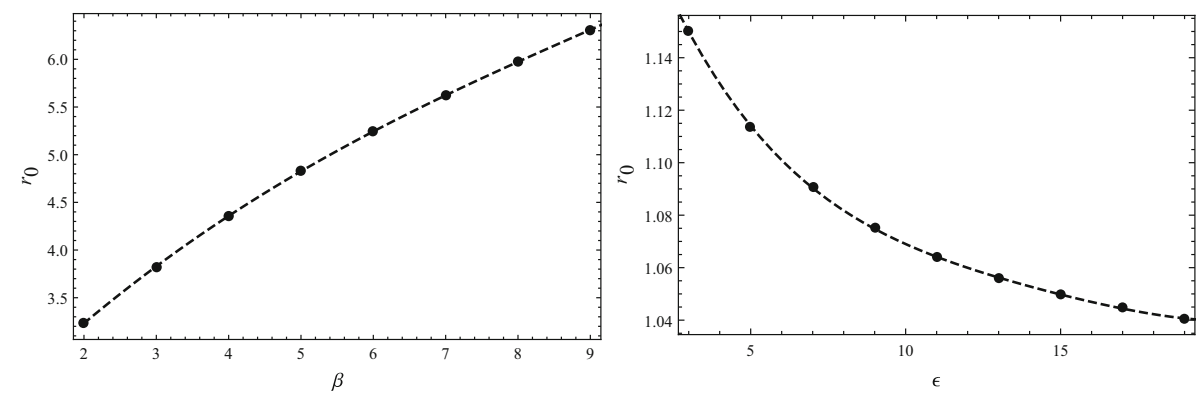

Fig. 1 Interpolations of $r_{0}$ vs $\beta$ and of $r_{0}$ vs $\epsilon$. The curves have been obtained through Wolfram Mathematica and represent an extrapolation based on the fitting functions $3.03 \cdot 10^{-5} x^{4}+1.13 \cdot 10^{-3} x^{3}-4.65 \cdot 10^{-2} x^{2}+$ $8.01 \cdot 10^{-1} x+1.79$ and $2.43 \cdot 10^{-6} x^{4}-1.43 \cdot 10^{-4} x^{3}+3.30 \cdot 10^{-3} x^{2}-3.80 \cdot 10^{-2} x+1.24$ respectively for the left and right plots

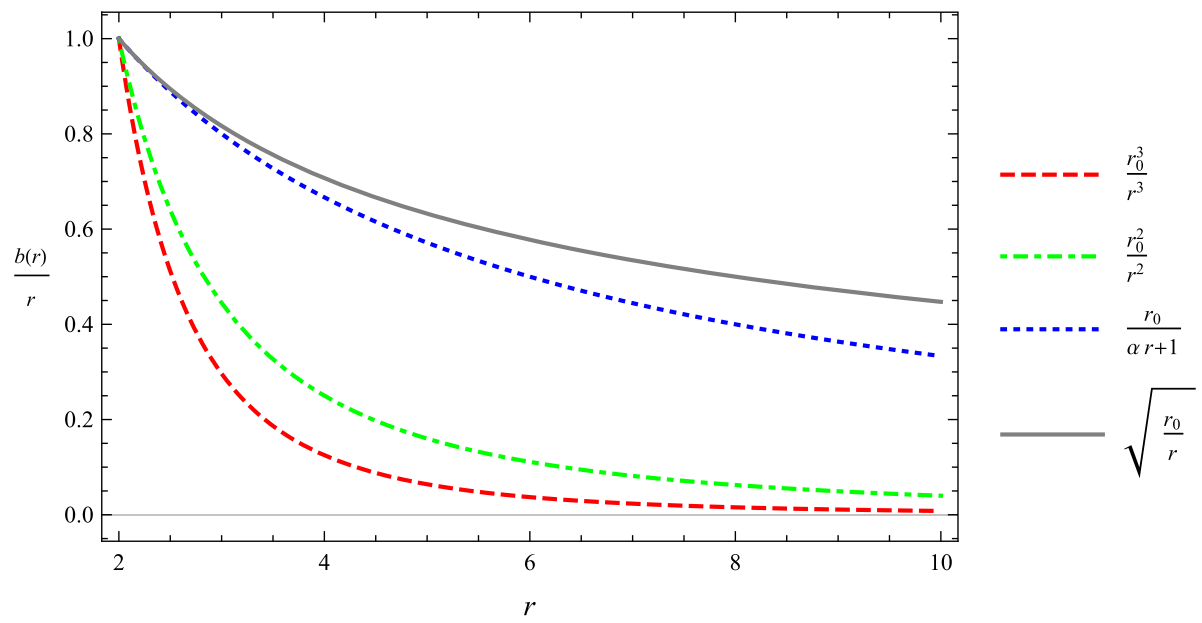

Fig. 2 Comparison between the shape functions of our models (red and blue lines), the standard approach proposed in [8] (grey line) and the model proposed in [28] (green line). The value of the $\beta$ parameter chosen for our model (4a) is $\beta=2$. Our Padé expansion better adapts to the standard approach than other phenomenological ansatz

\subsection{Theoretical considerations}

We assumed a power-law form for $f(R)$, where GR is recovered for $\epsilon=0$. From our analyses, it is possible to provide two classes of the shape function $b(r) / r$. The first possibility, already adopted in the literature, represents a class of inverse powers with respect to $r$ [27,28]. This approach departs significantly from the one provided in the original work by Morris and Thorne [8] as it appears evident from Fig. 1. Even though appealing, these possibilities are therefore disfavored than the Padé expansion that we proposed above. The $(0,1)$ Padé polynomial resembles much more the Morris-Thorne shape function and candidates as a suitable approach that turns out to be model-independent in reconstructing $b / r$. The expansion of $b / r$ is constructed by means of rational series. The only dependence from the model occurs as one chooses the order $(n, m)$. This approach is significantly better than ad hoc functions postulated at the beginning over $b$. In this respect, it is possible to provide two 
cases summarized in Tables 1 and 2. In Table 1, we consider the case of inverse power law approximation. It appears evident that the case $f \sim R^{2}$ is not recovered indicating small departures from the Starobinsky scalaron model [19]. The inverse solution, i.e., $1+\epsilon$ negative, is not excluded. In this case, the repulsive effects are stronger than the case of positive $1+\epsilon$. On the other hand, the Padé approximation excludes GR as well as the previous case but shows very small departures from $r_{0}$, indicating moreover that the Starobinsky scalaron is excluded again. In particular, the energy conditions are not fulfilled in the case of odd $\epsilon$. The results are well-suited in the Padé scenario and candidate to reconstruct the shape function without imposing any ad hoc functions. In all the aforementioned cases, it is possible to notice that $\epsilon$ is quite small, confirming that only small deviations from GR are permitted as soon as one considers wormholes in extended theories of gravity with vanishing sound speed (Fig. 2).

\section{Final outlooks and perspectives}

In GR, wormhole solutions are possible only if exotic matter is considered. In other words, standard matter prevents the wormhole stability and, consequently, its formation. Hence, natural landscapes in which wormholes may exist could be represented by extended and/or modified theories of gravity. There, the wormhole structure can be traversable without considering exotic matter contributions, i.e., a fluid with a negative energy and pressure density violating the energy conditions.

Here, we considered $f(R)$ gravity. Postulating a power-law form $f(R)=f_{0} R^{1+\epsilon}$, we investigated two possible approaches to characterize the shape function and, in particular, the ratio $b(r) / r$. The first attempt is a phenomenological inverse power law, recovered from widely investigated approaches in the literature. The second considers the numerical pathology of the ratio $b(r) / r$ within the throat. We thus introduced the Padé approximant to characterize the shape function in a model-independent way. Our strategy to decide the Padé orders is straightforward: we singled out the simplest approximant that resembles a first-order Taylor expansion. To do so, there are two possibilities, i.e., the $(1,0)$ and $(0,1)$ expansions. The first coincides with pure Taylor expansion and is unable to guarantee that $b \rightarrow 0$ as $r \rightarrow \infty$. The second possibility, namely $(0,1)$ fulfills our requirement. Thus, we worked out the wormhole solution under this ansatz, adding the additional requirement of stable fluids, whose perturbations are negligible inside the throat. For this purpose, we assumed the sound speed to vanish in analogy to cosmological contexts where the sound speed is associated with the fluid evolution. In particular, the sound speed has to vanish on the wormhole throat. Finally, it is possible to constrain the set of coefficients $\left(\beta, \epsilon, r_{0}\right)$. It is worth noticing that in all the analyzed cases, as soon as GR is recovered, exotic matter is needed to satisfy wormhole stability and traversability criteria.

In future works, we will extend such a scenario considering a general Padé approach to characterize the shape function. Other extended/modified theories of gravity will be taken into account besides $f(R)$ gravity.

Acknowledgements This work was partially supported by the Ministry of Education and Science of the Republic of Kazakhstan, Grant: IRN AP08052311. SC and LM acknowledge the partial support of Istituto Nazionale di Fisica Nucleare (INFN) iniziative specifiche MOONLIGHT2 and QGSKY.

Funding Open Access funding provided by Università degli Studi di Napoli Federico II. 
Open Access This article is licensed under a Creative Commons Attribution 4.0 International License, which permits use, sharing, adaptation, distribution and reproduction in any medium or format, as long as you give appropriate credit to the original author(s) and the source, provide a link to the Creative Commons licence, and indicate if changes were made. The images or other third party material in this article are included in the article's Creative Commons licence, unless indicated otherwise in a credit line to the material. If material is not included in the article's Creative Commons licence and your intended use is not permitted by statutory regulation or exceeds the permitted use, you will need to obtain permission directly from the copyright holder. To view a copy of this licence, visit http://creativecommons.org/licenses/by/4.0/.

\section{References}

1. M. Visser, Lorentzian Wormholes: From Einstein to Hawking (AIP Press, London, 1996)

2. D.C. Dai, D. Stojkovic, Phys. Rev. D 100, 083513 (2019)

3. J. Maldacena, L. Susskind, Fortsch. Phys. 61, 781 (2013)

4. J. Gratus, P. Kinsler, M.W. McCall, Found. Phys. 49, 330-350 (2019)

5. A. Ovgun, K. Jusufi, I. Sakalli, Phys. Rev. D 99, 024042 (2019)

6. K. Jusufi, A. Ovgun, Phys. Rev. D 97, 024042 (2018)

7. A. Einstein, N. Rosen, Phys. Rev. 48, 73-77 (1935)

8. M.S. Morris, K.S. Thorne, Am. J. Phys. 56, 395 (1987)

9. S. Capozziello, F.S.N. Lobo, J.P. Mimoso, Phys. Rev. D 91 (2015)

10. M. Li, X.D. Li, S. Wang, Y. Wang, Front. Phys. 8, 828-846 (2013)

11. E.J. Copeland, M. Sami, S. Tsujikawa, Int. J. Mod. Phys. D 15, 1753 (2006)

12. D. Hochberg, T.W. Kephart, Phys. Rev. Lett. 70, 2665-2668 (1993)

13. K. Bamba, S. Capozziello, S. Nojiri, S.O. Odintsov, Astrophys. Space Sci. 342, 155 (2012)

14. S. Capozziello, M. De Laurentis, Phys. Rept. 509, 167-321 (2011)

15. S. Capozziello, V.F. Cardone, A. Troisi, JCAP 0608, 001 (2006)

16. S. Capozziello, R. D’Agostino, O. Luongo, Int. J. Mod. Phys. D 28, 101930016 (2019)

17. T.P. Sotiriou, V. Faraoni, Rev. Mod. Phys. 82, 451-497 (2010)

18. I.L. Buchbinder, S.D. Odintsov, I. Shapiro, Effective Action in Quantum Gravity (IOP Publishing, Bristol, 1992)

19. A.A. Starobinsky, Phys. Lett. B 91, 99 (1980)

20. T.P. Sotiriou, V. Faraoni, S. Liberati, Int. J. Mod. Phys. D 17, 399 (2008)

21. A. De Felice, S. Tsujikawa, Living Rev. Rel. 13, 3 (2010)

22. S. Capozziello, Int. J. Mod. Phys. D 11, 483 (2002)

23. S. Capozziello, A. Stabile, A. Troisi, Class Quantum Grav. 25, 085004 (2008)

24. S. Capozziello, C.A. Mantica, L.G. Molinari, Int. J. Geom. Methods Mod. Phys. 16, 1950008 (2018)

25. S. Capozziello, C.A. Mantica, L.G. Molinari, Gen. Relat. Gravit. 52, 36 (2020)

26. V. De Falco, E. Battista, S. Capozziello, M. De Laurentis, Phys. Rev. D 101, 104037 (2020)

27. S. Capozziello, T. Harko, T.S. Koivisto, F.S.N. Lobo, G.J. Olmo, Phys. Rev. D 86, 127504 (2012)

28. J.L. Rosa, J.P.S. Lemos, F.S.N. Lobo, Phys. Rev. D 98, 064054 (2018)

29. C. Gruber, O. Luongo, Phys. Rev. D 89, 103506 (2014)

30. H. Wei, X.P. Yan, Y.N. Zhou, J. Cosm. Astrop. Phys. 1401, 045 (2014)

31. S.G. Krantz, H.R. Parks, A Primer of Real Analytic Functions (Birkhäuser, London, 1992)

32. A.V. Astashenok, S. Capozziello, S.D. Odintsov, V.K. Oikonomou, Phys. Lett. B 811, 135910 (2020)

33. S. Capozziello, M. De Laurentis, R. Farinelli, S.D. Odintsov, Phys. Rev. D 93, 023501 (2016)

34. H. Padé, Ann. Sci. Ecole Norm. Sup. 9, 3-93 (1982)

35. V. Nestoridis, J. Contemp. Math. Anal. 47, 168-181 (2012)

36. M. Della Morte et al., JHEP 1203, 055 (2012)

37. D. Hochberg, M. Visser, Phys. Rev. D 58, 044021 (1998)

38. M.S. Linton, A. Pourtsidou, R. Crittenden, R. Maartens, JCAP 04, 043 (2018)

39. S. Capozziello, M. De Laurentis, O. Luongo, A.C. Ruggeri, Galaxies 1, 216-260 (2013)

40. O. Luongo, M. Muccino, Phys. Rev. D 98, 103520 (2018)

41. Farbod Hassani, Benjamin L'Huillier, Arman Shafieloo, Martin Kunz, Julian Adamek, JCAP 04, 039 (2020)

42. M. Kunz, Phys. Rev. D 80, 123001 (2009)

43. O. Luongo, H. Quevedo, Int. J. Mod. Phys. D 23, 1450012 (2014)

44. K. Boshkayev, T. Konysbayev, E. Kurmanov, O. Luongo, M. Muccino, Galaxies 8, 74 (2020)

45. S. Capozziello, P. Jovanovic, V. Borka Jovanovic, D. Borka, JCAP 1706, 044 (2017) 
46. S. Capozziello, P. Martin-Moruno, C. Rubano, Phys. Lett. B 664, 12 (2008)

47. S. Capozziello, R. D'Agostino, O. Luongo, Gen. Relat. Gravit. 51(1), 2 (2019)

48. M. Calza, A. Casalino, O. Luongo, L. Sebastiani, Eur. Phys. J. Plus 135(1), 1 (2020) 